\title{
AIAA 01-3204
}

Shock Waves Mitigation at Blunt Bodles using Needles and Shells against a Supersonic Flow

M. Glinsky

Hamp ton University, USA

I.M. Blankson

NASA Glenn Research Center, USA

VI. Salkharov

and

AI. Shvets

Institute of Mechanics/Moscow State University, Russia

$3^{\text {क }}$ AIAA/ASME/SAE/ASEE Joint Propulsion Conference July 8-11, 2001/ Salt Lake CIty, UT

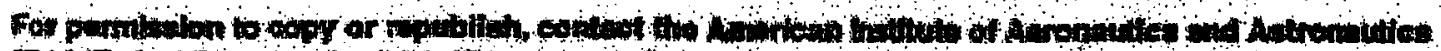

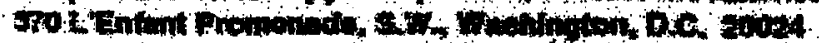




\title{
Shock Waves Mitigation at Blunt Bodies Using Needles and Shells against a Supersonic Flow.
}

\author{
M. Gilinsky ${ }^{*}$ \\ Hampton University, USA \\ I.M. Blankson \\ NASA Glenn Research Center, USA \\ V.I. Sakharov*** \\ and \\ A.I. Shvets**** \\ Institute of Mechanics/Moscow State University, Russia
}

\begin{abstract}
The paper contains some experimental and numerical simulation test results on cylindrical blunt body drag reduction using thin spikes or shell mounted in front of a body against a supersonic flow. Experimental tests were conducted using the Aeromechanics and Gas Dynamics Laboratory facilities at the Institute of Mechanics of Moscow State University (IM/MSU). Numerical simulations utilizing NASA and IM/MSU codes were conducted at the Hampton University Fluid Mechanics and Acoustics Laboratory. The main purpose of this research is to examine the efficiency of application of multiple spikes for drag reduction and flow stability at the front of a blunt body in different flight conditions, i.e. Mach number, angle of attack, etc. The principal conclusions of these test results are: multiple spike/needle application leads to decrease of drag reduction benefits by comparison with the case of one central mounted needle at the front of a blunt body; but increase lift benefits. The research was conducted under the NASA Faculty Research Award for Hampton University, Hampton, VA, grant \# NAG-3-2422, grant \# NAG-32495, and under the Civil Research Development Foundation (CRDF) grant, \# RE1-2068, for joint research between the NASA Glenn Research Center and Institute
\end{abstract}

\footnotetext{
* Research Professor, Senior Member AIAA, USA

** Senior Scientist, AIAA Associate Fellow, USA

*** Leading Senior Scientist, Russia

**** Professor, IM/MSU Laboratory Chief, Russia
}

of Mechanics of Moscow State University, Russia.

\section{INTRODUCTION}

A thin spike mounted in front of a blunt body can be used to decrease the drag and the heat transfer in a high oncoming flow velocity. The spiked blunt body at an attack angle decreases not only the drag-although not so effectively as the case of zero attack angle-but also increases the lift. Therefore, a nose spike may be used for take-off and other flight conditions. By varying the length and diameter of the protruding probe (spike or needle), flow characteristics can be controlled. The thin straight probe is a handy and simple control device, compared to variable geometry skirts. However, its applicability is somewhat limited, unless a proper arrangement is made to eliminate the unfavorable effect of flow oscillation on the probe, which may cause an aerodynamic disturbance. The summary of the early experimental and theoretical papers where flow past a body with a spike was studied is contained in the well-known book by P.K. Chang (1970) and in the Russian book [1]. Six regimes of supersonic flow past a cylinder with conical nose and a spike protruding from it are distinguished for specified Mach and Reynolds numbers of the oncoming flow which includes the regime of stationary flow with drag reduction and the regimes of unsteady pulsating and fluctuating flows. 
Since 1950 , the application of spike-nosed blunt bodies has drawn the attention of scientists. In the USA and the former USSR, a number of researches were conducted in this area. In the first group of experimental tests, the main aerodynamic characteristics of different blunt body shapes with needles were obtained. In the following stage, numerical simulations of this problem were conducted. Significant attention was directed to experimental and numerical tests of different oscillatory regimes of the flow at the spikenosed blunt bodies that were observed in some range of needle length values.

The main cause of this phenomenon is connected with flow separation at the needle or body points of some essential curvature changes. In the common case, the flow is not always steady, and may be periodically unsteady. Two distinct modes of instability have been observed. In the "pulsation" mode, the conical separation bubble formed on the concave part of the body periodically inflates and expands in the radial direction, taking a hemispherical shape. In the "oscillation" mode, a conical foreshock envelops the separation bubble and the accompanying shear layer oscillates laterally. Its shape changes periodically from concave to convex. The pulsation mode was first observed by Mair [2] and the oscillation mode was observed by Bogdonoff and Vas [3]. The terminology is due to Kabelitz (1971). A comparison shows that the oscillating flow around a concave body is a sub-case of the self-sustained oscillations of impinging free shear layers reviewed by Rockwell and Naudascher [4].

In the next sections, we will present the main experimental tests and numerical simulation results recently obtained by the authors in this area with the purpose of improving the efficiency of some propulsion systems. Experimental tests were conducted in the IM/MSU aerodynamic wind tunnel, A-7 [5], and numerical simulations were conducted at the Hampton University FM\&AL during the visit of two IM/MSU scientists to the USA for joint research.

\section{EXPERIMENTAL SETUP}

The main purpose of this research is to estimate the influence of the application of several needles mounted at the front of a blunt body against a supersonic flow by comparison with the traditional one needle application. Experimental tests were conducted using an axisymmetic cylindrical model with a flat forward part, i.e. butt-end, of diameter, $\mathrm{D}=80 \mathrm{~mm}$. Two types of models were tested: a) with 1 needle $(\mathrm{n}=1)$ and $b)$ with 5 needles $(n=5)$. In both cases, one central needle was mounted at the center of the circular front flat part of radius, $R=40 \mathrm{~mm}$. For the second series of models, another 4 needles were placed symmetrically around the central needle. They were placed at the angular interval of $90^{\circ}$ and at the radial distance, $r=0.5 \mathrm{R}$. Nondimensional needle lengths, $L / D$, were chosen based on constructive opportunities of application, and on known referenced data in this field. These data show that maximum drag reduction using needles at supersonic speeds can be achieved in the range of needle lengths, $\mathrm{L} / \mathrm{D}=1.5-2.0$, so that three needle lengths were manufactured and tested: $\mathrm{L} / \mathrm{D}=1.5,1.0$, and 0.5 . For this needle length range, the end of the needle at one limit, $\mathrm{L} / \mathrm{D}=1.5$, is located farther from the butt-end than the detached bow shock wave which occurred in the case of flow without a needle at the body front. For the other limit, $L / D=0.5$, the needle is located completely inside the compression shock layer, i.e. closer to the butt-end than the corresponding detached bow shock wave. Before the experimental tests, we intended to manufacture only one needle set with needle thickness (diameter), $\mathrm{d}=0.1 \mathrm{D}$. However, during the first experiments, it became obvious, that in this case, $n=5$, the needle-buttend model too thick was and creates the single 
shock wave in front the spikes. On the other hand, needle geometry (thickness, length, shape) can play the main role in the organization of a flow circuit at the body. It was assumed that when the forward circuit separation zone forms at the single needle, the shock wave has conical or ogival shape. For several needles, another shock wave geometry should appear, similar to the case of supersonic flow around star-shaped bodies. Namely, with Mach type or regular interaction of shock waves, similar to a flow around several forward separation zones, or to formation of a uniform forward separation zone. As a result of this analysis, the following needle geometric parameters were chosen: $\mathrm{D} / \mathrm{L}$ $=0.05 ; 0.81 ; 0.1$, i.e. for the given model size, the needle diameters are: $d=4 ; 6.5$; and $8 \mathrm{~mm}$.

A supersonic wind tunnel with permeable test section wall was employed for the experimental tests. This wind tunnel has the transonic speed range with continuous transition through to supersonic speeds. The wind tunnel with test section size, $0.6 \times 0.6 \mathrm{~m}$, is equipped a pressure and suction ejector allowing the conduction of experimental tests in a wide range of Mach number, $M=0,4-4,0$ and Reynolds number, $\operatorname{Re}=1 \times 10^{5}-2.5 \times 10^{7}$, where Reynolds number is calculated relative to $1 \mathrm{~m}$. The aerodynamic forces acting on the model are measured on a four-component tenzometric scale of the $19 \mathrm{~mm}$ diameter TV128 type. A tenzoamplifier device is a tracking system controlling a voltage value from the measuring diagonal tenzometric scale that is cemented on the elastic elements of the model. Estimation of errors has shown that the total root-mean-square measurement error for the aerodynamic coefficients are $\left\langle\mathrm{C}_{\tau}\right\rangle=0.03$ for the axial force coefficient (if $\alpha=0^{\circ}$ then $\mathrm{C}_{\tau}=\mathrm{C}_{\mathrm{D}}$ ), for the normal force coefficient, $<\mathrm{Cn}>=0.02$, and for the longitudinal moment coefficient, $<\mathrm{m}_{\mathrm{z}}>=0.02$.
The pressure gauges IKD-27 were employed for measurement of the free stream total pressure. Static and total pressures were also measured. The relative root-mean-square error of pressure measurement $\langle p\rangle$ was $\sim 0.01$ at the upper measurement limit. Automatic processing of these measurements was carried out by the computer complex and printed. The Mach number in the test area of the wind tunnel is close to three, $M=2.97$, and Reynolds number, $\mathrm{Re}=1.6 \times 10^{6}$, wherc Reynolds number is calculated on the free stream parameters and model diameter. Two types of visualizations of the stream were used: a) with large exposure time, $t=0.01 \mathrm{sec}$, that considerably exceeds the duration of a pulsation cycle. These depict average flow field features in time. And b) with small exposure time, $t=10^{-6} \mathrm{sec}$, that allows the observation of flow fluctuations and shock wave movement.

\section{EXPERIMENTAL RESULTS}

3.1 It is known that for supersonic gas flow with Mach number, $M=3$, around the cylindrical butt-end, the pulsation regime that first was observed by Mair [2], occurs in a range of needle lengths about $\mathrm{L} / \mathrm{D}=0.4-1.7$ (Maull [6]). In this case, pulsations of "the second sort" (Antonov, Shalaev [7]) take place. The body shape is the determining factor as to which pulsations occur. Therefore, for the butt-end model with only one needle, such pulsations could be expected in the present experiments. In Mair's experiments, the pulsation frequency was $6 \times 10^{3} \mathrm{sec}^{-1}$ for Mach number, $M=1.96$, and in Maull's experiments, for Mach number, $M=6.8$, it was $2 \times 10^{4} \mathrm{sec}^{-1}$. In the present methodical set of experiments the pulsation regimes were also observed: different pulsation phases were seen on the shlieren photos of the flow field and measured forces had oscillatory character as shown by the tenzoscales. A consecutive registration of forces at the specific frequencies was impossible because the measuring instrument was too inertial. 
However, some estimations of the fluctuation ranges for various force components were obtained.

3.2 All experimental test results presented in this paper were obtained in the IM/MSU wind tunnel for fixed free stream Mach number, $M=3$. In Figures 1a-f, the drag coefficient, $C_{D}$, vs different geometric parameters of the needles are shown for a zero attack angle, $\alpha=0^{\circ}$. In Figure $1 \mathrm{a}$, the $\mathrm{C}_{\bar{D}}$ coefficient is plotted for the case $M=3, n=1, d / D=0.082$, $L / D=1$, and $\alpha=0^{\circ}$. These values were measured during one experiment with the equal time interval ( $\mathrm{k}$ is a registration number). One can see that there is an oscillatory change of $C_{D}$ coefficient that reflects the existing pulsation range qualitatively. The tenzoscales employed can not register each fluctuation cycle (the scales own fluctuation frequency is near 100 $\mathrm{Hz}$ ). Therefore it does not show exact values of forces in the extremal points of the fluctuations. Nevertheless, when there are not regular fluctuations as in the case of a cylindrical butt-end without any needle, for example, the measured $C_{D}$ values during one experiment change not more than $\sim 0.03>$, i.e. within the scales error of measurement. Comparison of fluctuation results for two models with 1 and 5 needles shows that in the last case $(n=5)$, the $C_{D}$ deviations from its average value are much less than for a single needle $(n=1)$.

In Figure $1 \mathrm{~b}$ the average values of the $C_{D}$ coefficient (black symbols) and its oscillatory values (white symbols) are shown for the model with 5 needles $(n=5)$ with different lengths, $\mathrm{L} / \mathrm{D}=0.0 ; 0.5 ; 1.0 ;$ and 1.5 and different needle diameters, $\mathrm{d} / \mathrm{D}=0.05,0.082$, and 0.1 . For the same needle diameters, this coefficient value vs needle number is illustrated in Figure 1c for the needle length, $D / L=1$. Comparison of $C_{D}$ coefficient for single needle $(n=1)$ and five needle $(n=5)$ models vs needle length is shown in Figure 1d, and vs needle diameter for different needle lengths is shown in Figure 1e. In the last figure, experimental data obtained by Hunt for a single needle are also plotted. Comparison of this coefficient for single needle and five needle models vs needle length for the needle diameter, $d / D=0.082$ is illustrated in Figure 1f. Average values for different force coefficients, drag- $C_{D}$, normal- $C_{N}$, and longitudinal momentum relative to the point, $\mathrm{x}=1$, vs angle of attack, $\alpha$, are shown in Figures 2a-c for the single needle model, and in Figures $2 \mathrm{~d}-\mathrm{f}$, for the 5-needle model.

3.3 Several additional experimental tests were conducted with the purpose to determine the influence of multi-needle $(n>5)$ application to the force coefficients. Figures 3a-d. The limit case for infinite needle number is illustrated in Figure 3a,b. The drag coefficient for the solid shell mounted to the front butt-end by the 5 needles is plotted vs shell length (a) and angle of attack (c). In Figure 3b, this coefficient is shown for large finite needle number, $n=53$ and $n=103$. These needles are mounted symmetrically along the coincident circles located from the circle center on the radial distances: $\mathrm{R}=0 ; 5 ; \ldots 35 \mathrm{~mm}$. Recall that the butt-end circle diameter is $\mathrm{D}=80 \mathrm{~mm}$. A drag coefficient comparison for all considered cases, $\mathrm{n}=1 ; 5 ; 53$ and 103, is shown in Figure $3 \mathrm{~d}$ that illustrates a preferable application for drag reduction of single needles.

3.4 Optical methods of flow visualization were employed for better understanding of this phenomenon and for explanation of the force aerodynamic characteristics changes.

They include a traditional shlieren method and high resolution video filming of the unsteady pulsation regimes. In Figure 4a-I, the main models tested in supersonic flow with Mach number, $M=3$, at zero angle of attack are shown: a)-butt-end without needles; b),c) -with single needle for two different instants of pulsation flow regime; d)-f) -for 5 thin needles 
model of thickness, $\mathrm{d} / \mathrm{D}=0.05$, and different needle lengths, $\mathrm{L} / \mathrm{D}=0.5 ; 1.0$ and 1.5 ; and $\mathrm{g}$ )-i) -for 5 thick needles model of thickness, $\mathrm{d} / \mathrm{D}=0.1$ with the same as previous needle lengths.

\section{NUMERICAL SIMULATION RESULTS}

4.1 A finite-volume implicit numerical method was developed to solve the two-dimensional and axisymmetric time-dependent NavierStokes equations (NSE) in the conservative form. It was assumed that the flux vector could be split into 'viscous' and 'inviscid' parts. The solution of the Riemann problem of heatconducting gas with frozen chemical composition has been obtained for a computation of 'inviscid' fluxes. Spatial derivatives in the 'viscous' terms are approximated with second-order accuracy. Piecewise-parabolic distributions of the physical variables over the network cells and 'minmod' limiters lead to a TVD-scheme of second-order accuracy. The finite-difference equation set is resolved by the sweep method along the lines normal to the surface and the Gauss-Seidel iteration procedure. Coordinateoriented differences are introduced in the implicit part of the finite-difference operator. These are chosen in accordance with signs of the eigenvalues of Jacobi matrices in the convective terms. A new finite-difference scheme for solution of the viscous shock layer (VSL) equations with multicomponent diffusion and nonequilibrium chemical reactions in gas and on the body surface is developed due to the finite-volume approach. The flux-difference splitting method based on the linearized solution of the Riemann problem in isoenergetic approximation is applied for calculations of the inviscid fluxes through the interfaces of cells. Introducing into the expression for the fluxes limited anti-diffusion corrections provides realization of the TVD conditions and second order of accuracy of the smooth solution. The viscous fluxes are calculated with a space-centered scheme. The boundary conditions on the body surface and on the shock wave are approximated in the context of the general conception of a finitevolume method with the second order of accuracy. A steady solution of the difference equations is found by means of an iterative procedure based on solving pseudotimedependent VSL equations with an implicit scheme. The flowfield calculation at every time step is performed by the Gauss-Seidel line relaxation numerical technique. Based on this approach, the numerical code was developed by V. G. Gromov with colleagues $[8,9]$ at the IM/MSU for the MS platform and used for several research projects. Note that this approach is similar to the approach employed in the NASA numerical code VULCAN.

4.2 The purpose of numerical simulation of this problem is to check some experimental test results and to obtain more exact aerodynamic characteristics for pulsation flow regimes. Several numerical simulation tests were conducted for axisymmetric supersonic flow at the butt-end with the single needle (1model). Three examples of such test results are shown in Figures 5a-f where Mach (a-c) and pressure (d-f) has been plotted for the relatively thick needle, $\mathrm{d} / \mathrm{D}=0.1$, and different needle lengths, $\mathrm{L} / \mathrm{D}=0.9 ; 1.0$; and 1.6. These results were based on NSE simulation with the $\mathrm{k}-\omega$ turbulence model. In this case, steady solutions were obtained. One can see the presence of two separation zones at the corner points of the needle and main butt- end, as well as the vortical flow at the needle lateral surface. The drag coefficient calculated from the numerical simulation for the case tested experimentally, $\mathrm{d} / \mathrm{D}=0.1 ; \mathrm{L} / \mathrm{D}=1.0$, is equal to $C_{D}=0.52$ that is very close to the experimental value.

However, these numerical simulation results are very sensitive to changes of numerical scheme parameters or applied models. For 
example, for the same conditions in inviscid flow (Euler approximation) or for a laminar viscous flow, numerical simulation results show periodic oscillatory variation of the flow. An analysis of such solution leads to the conclusion that mass flow rate pulsation takes place. Such pulsatile flow at the needle is shown in Figure 6a-n for the free stream Mach number, $M=3$. In these pictures, Mach contours are plotted for different instants and show different shock wave positions and flow structure during one oscillation cycle.

\section{CONCLUSIONS}

As a result of these experimental and numerical simulation tests, the main conclusions were made. 1) Application of 5 needles instead of a single needle mounted at the front butt-end leads to growth of all force coefficients at zero angle of attack of the tested model as well as at almost all angles of attack, $\alpha<15^{\circ}$. Especially, such growth is observed for the relatively small needle lengths $(\mathrm{L} / \mathrm{D}=0.5)$ at zero angle of attack. 2) Increase of the needle number leads to some flow stabilization, i.e. oscillation amplitude decreases for the 5-needle model by comparison with the single needle model. 3) Normal force coefficient, $\mathrm{C}_{\mathrm{N}}$, for the 1 needle model is essentially less than for the 5-needle model, and this value grows almost monotonically with increase of angle of attack for the 5-model, and non- monotonically for 1model with maximum value at angle of attack, $\alpha \approx 10^{\circ}$.

\section{ACKNOWLEDGEMENTS}

We would like to acknowledge the NASA Glenn and Langley Research Centers, especially Dr. Dennis M. Bushnell, Charles W. McClinton, David W. Lam, and Curt Snyder from the Naval Air System Team for interest and support of our research. This research was partially conducted under the NASA grants: NAG-3-2422 and 2495, and under the supporting CRDF grant RE1-2068. We would like to thank Dr. Jay C. Hardin for his attention, interest in our research, review and useful suggestions.

\section{REFERENCES}

1. Shvets A.I., Aerodynamic of Supersonic Shapes, 1987, Moscow State University Press, Moscow.

2. Mair, W., "Experiments on Separated Boundary Layers on Probes in Front of Blunt Nosed Bodies in a Supersonic Air Stream," Philosophy Magazine, Vol. 43, 1952, pp. 695-716.

3. Bogdonoff S. M., Vas I. E. Preliminary investigations of spiked bodies at hypersonic speeds.- J. Aerospace Sci., 1959 , v. 26, № 2, p. 65-74.

4. Rockwell, D. and Naudasehar, E., "Review of SelfSustaining Oscillations of Flow Past Cavities,"

ASME Journal of Fluids Engineering. 100. 1978, pp. 152165.

5. Aerodynamic Facilities at the Institute of Mechanics MSU, 1985, MSU Publishing House.

6. Maull, D J, "Hypersonic Flow Over Axially Symmetric Spike Bodies," Journal of Fluid Mechanics, Vol. 8, Pt 4, Aug 1960, pp 584-594,

7. Antonov A. N. , Shalaev N. E. Some features of nonstationary separated currents on bodies with the established ahead needle. The mechanics of Fluid and Gas, 1979 , N 1, c. 97.

8. Levin V.A., Afonina N.E., Gromov V.G., NavierStokes Analysis of Supersonic Flow with Local Energy Deposition", AIAA-99-4967, Nov. 1999

9. Karlovskii, V.N., and Sakharov, V.I. , 1986, Numerical investigation of supersonic flow past blunt bodies with protruding spikes, 1986, Fluid Dynamics (Izv. Akad. Nauk SSSR - Mekh. Zhidk. Gaza), Vol.21, No.3, pp.437-445 

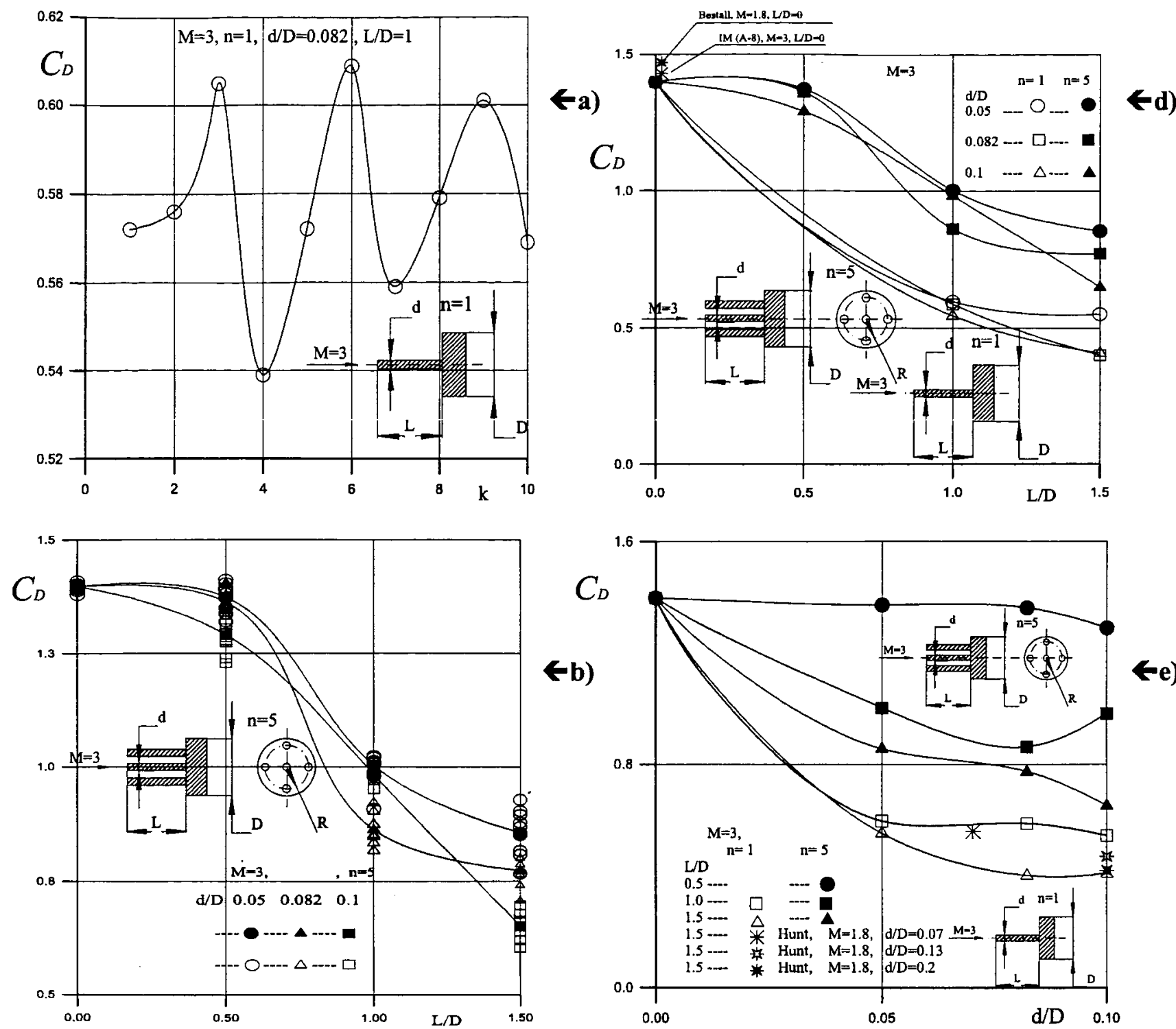

(-)

$C_{D}$

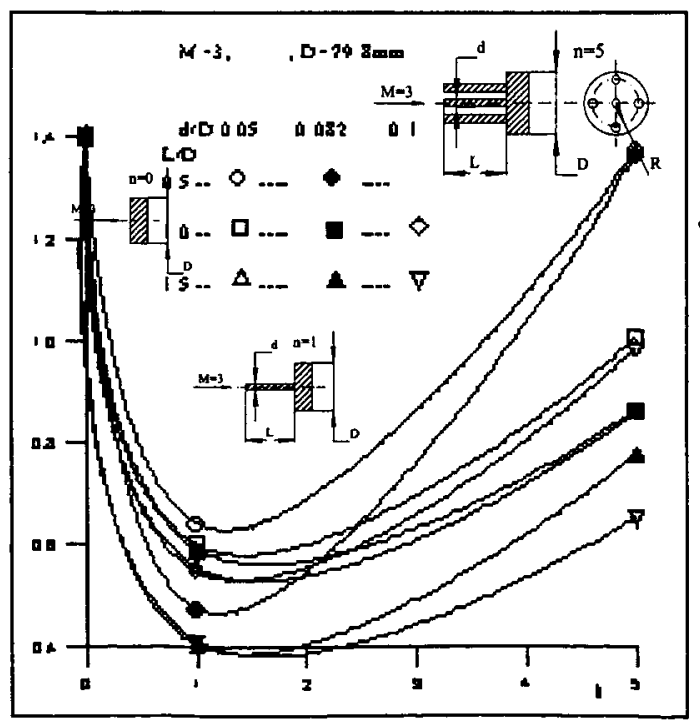

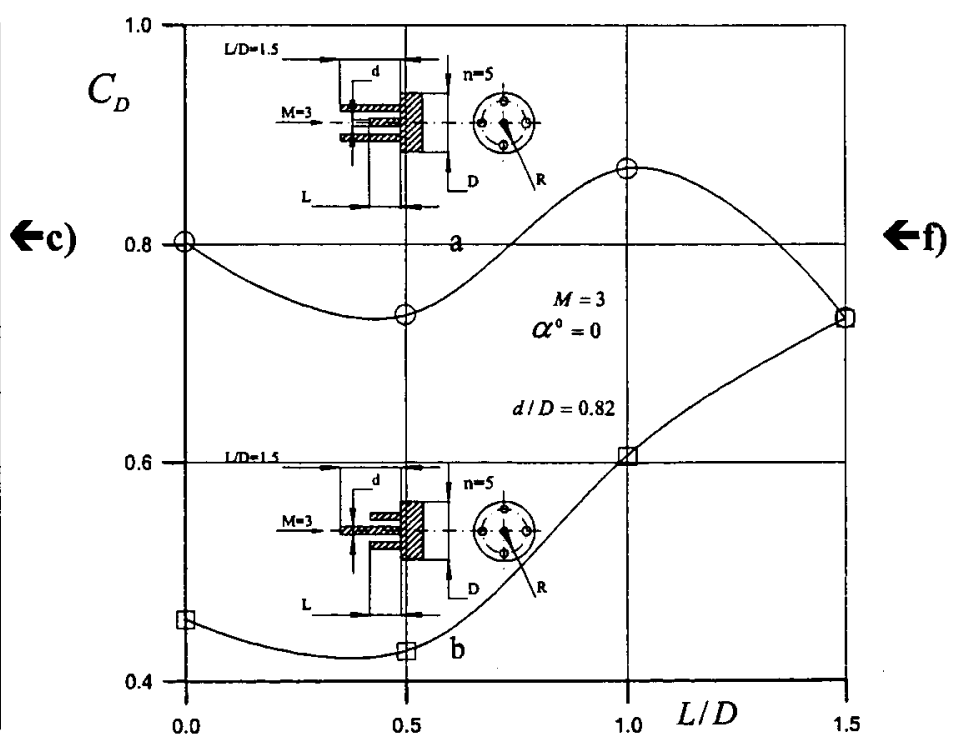

Fig.1 Drag coefficient, $C_{D}$, for 1 - and 5-needles butt-end models vs needle length, $L / D$, and diameter, $d / D ; D$ is circle diameter of front butt-end. 

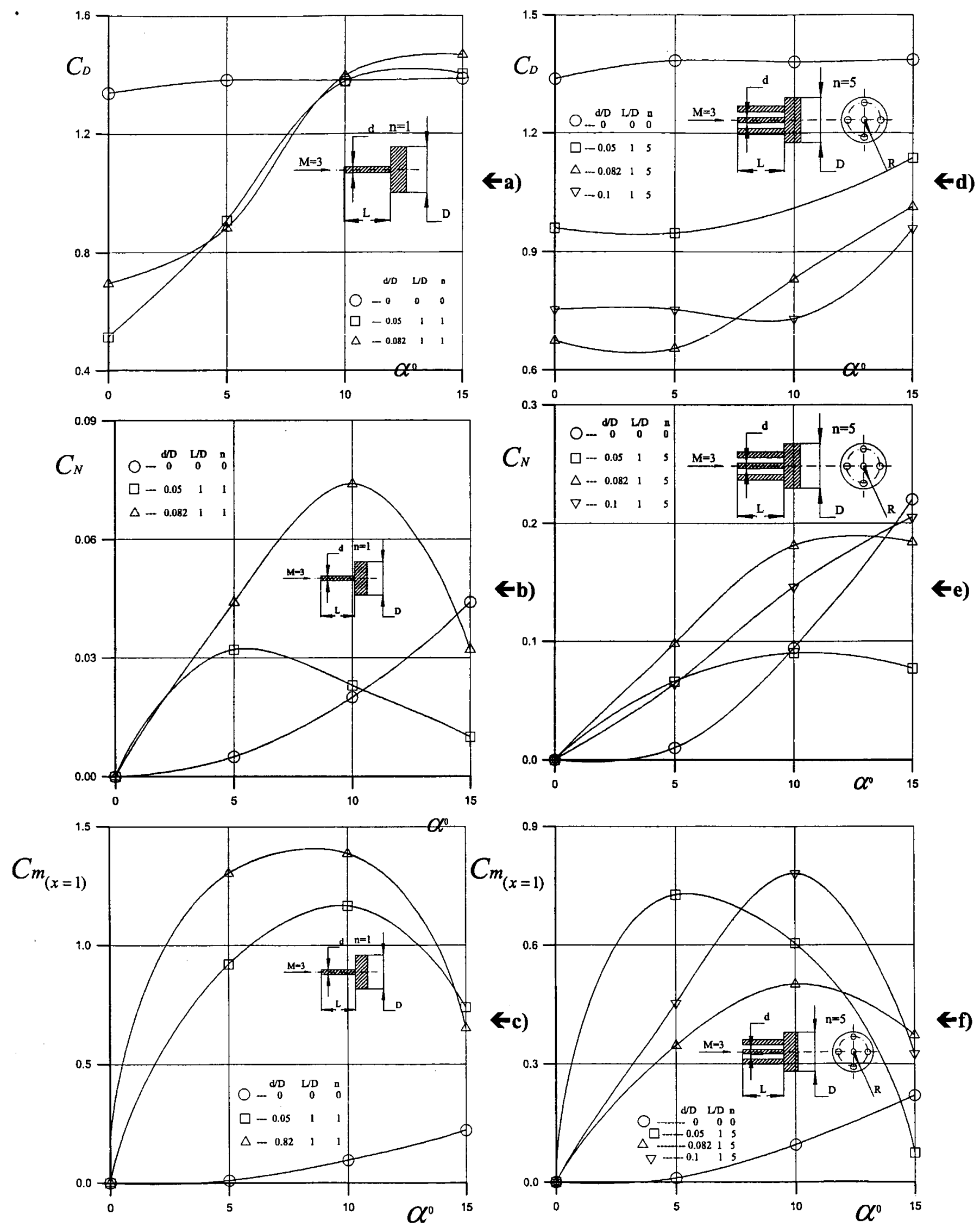

\&)

Fig.2 Aerodynamic characteristics of 1- and 5-needle butt-end tested at the IM/MSU Wind Tunnel A-7. 
$\mathrm{M}=3, \alpha=0, \mathrm{~L} / \mathrm{D}=0.5$

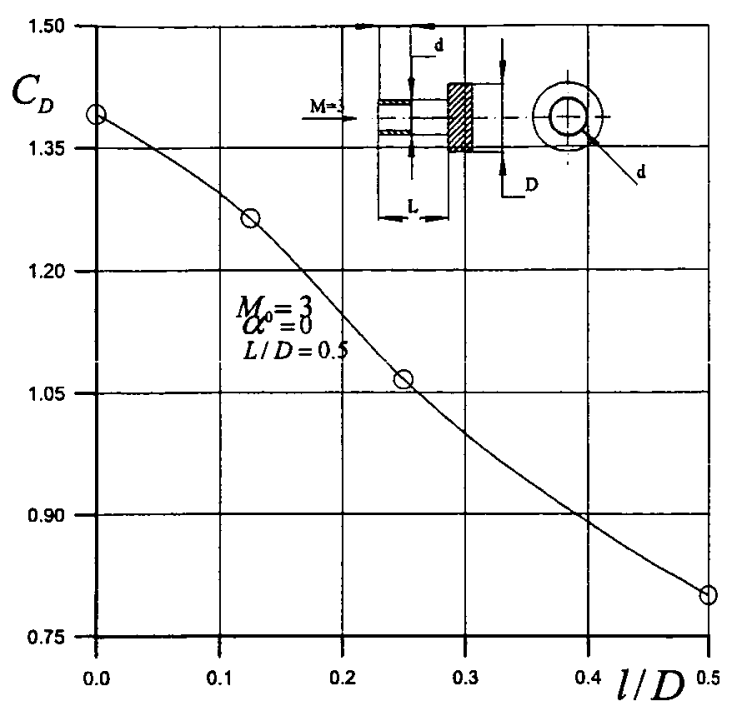

a)

$M=3, d / D=0.475$

$\begin{array}{lll} & \text { I/D } & \text { L/D } \\ \circ & -0 & -0 \\ \Delta & -0.125 & -0.5 \\ \nabla & -0.25 & -0.5 \\ \square & -0.5 & -0.5\end{array}$

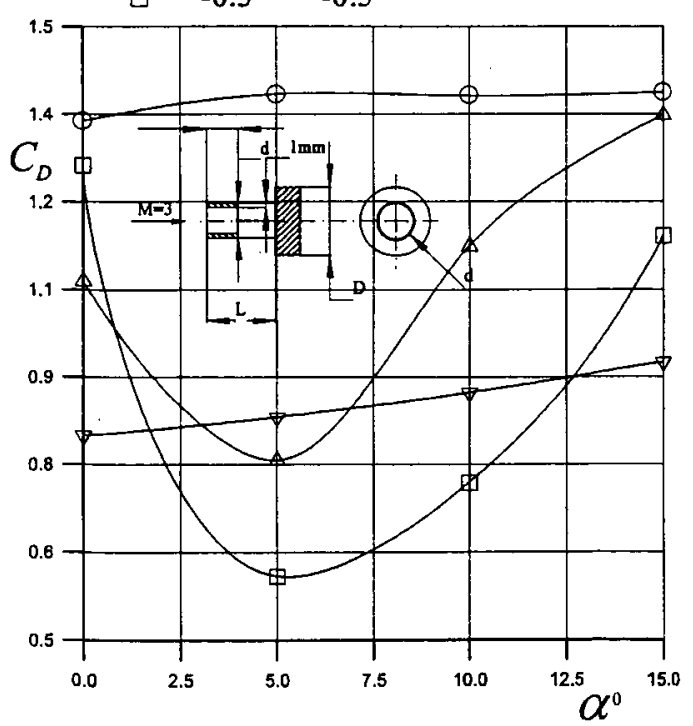

c)
$\mathrm{M}=3, \mathrm{~d} / \mathrm{D}=0.025, \mathrm{~L} / \mathrm{D}=0.438$

$\mathrm{n}=53 \mathrm{R}=0,5,15,25,35 \mathrm{~mm}$

$\mathrm{n}=103 \quad \mathrm{R}=0,5,10,15,20,25,30,35 \mathrm{~mm}$

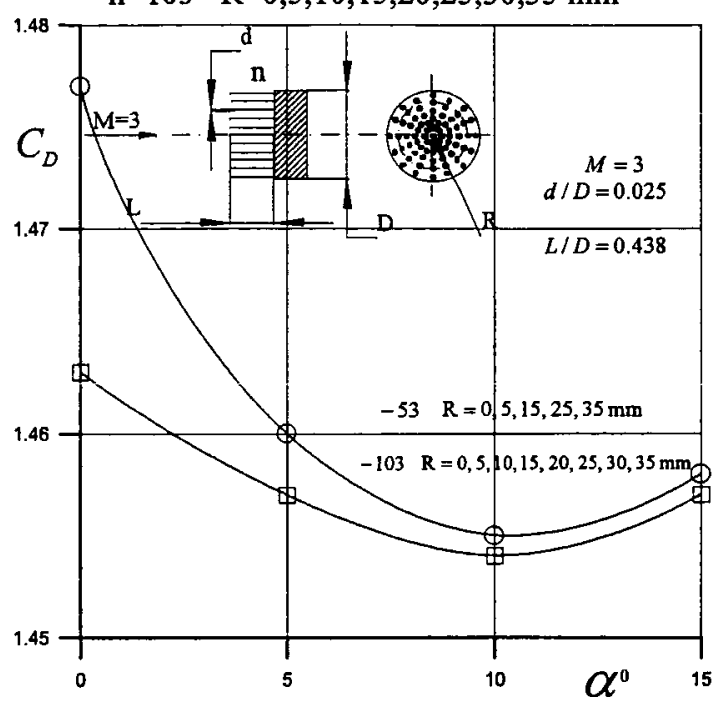

b)

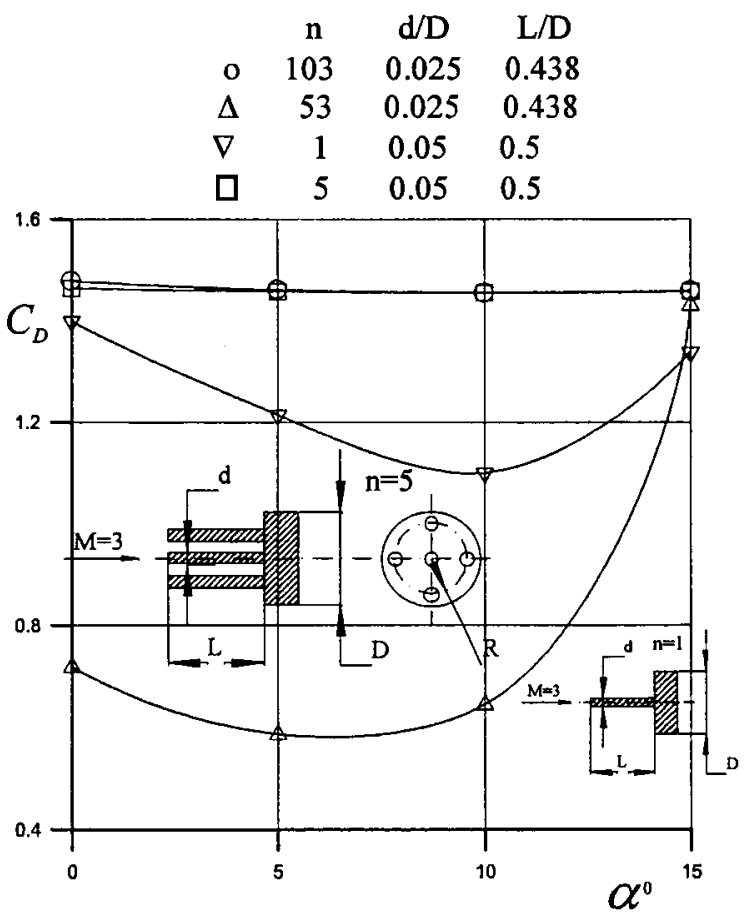

d) 


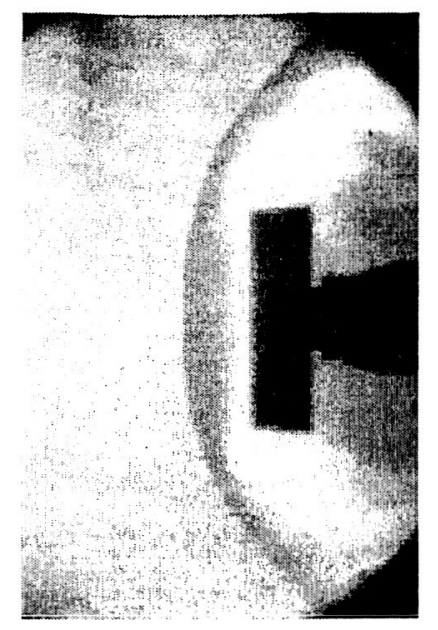

a) $-\mathrm{n}=\mathbf{0}, \mathrm{L} / \mathrm{D}=\mathbf{0}, \mathrm{d} / \mathrm{D}=\mathbf{0}$

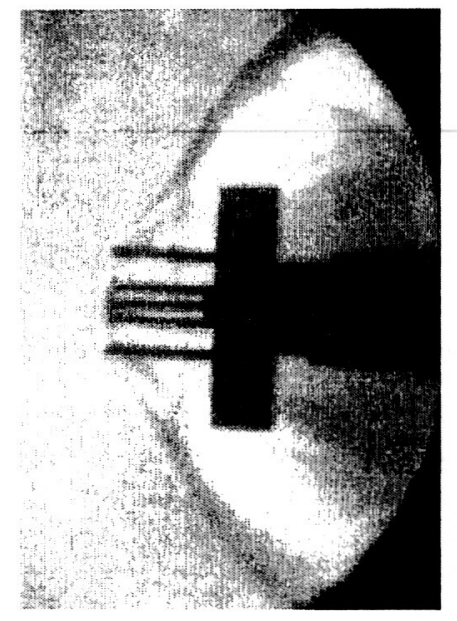

d)-n=5, L/D=0.5, d/D=0.05

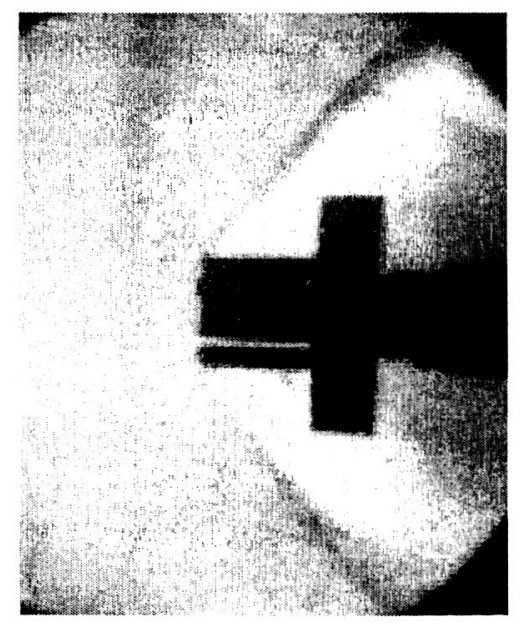
g) $-n=5, L / D=0.5, d / D=0.1$

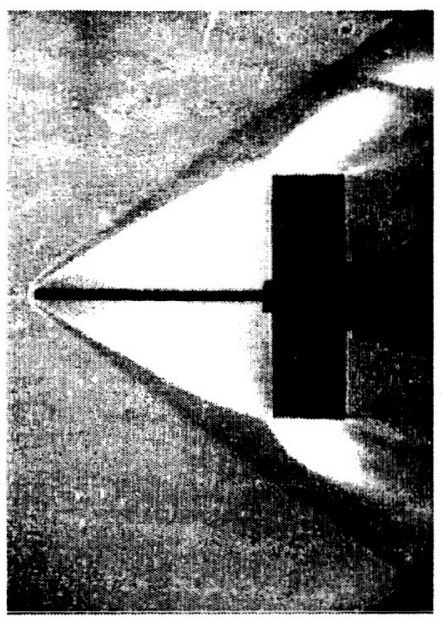

b)-n=1, L/D= 1, d/D=0.05

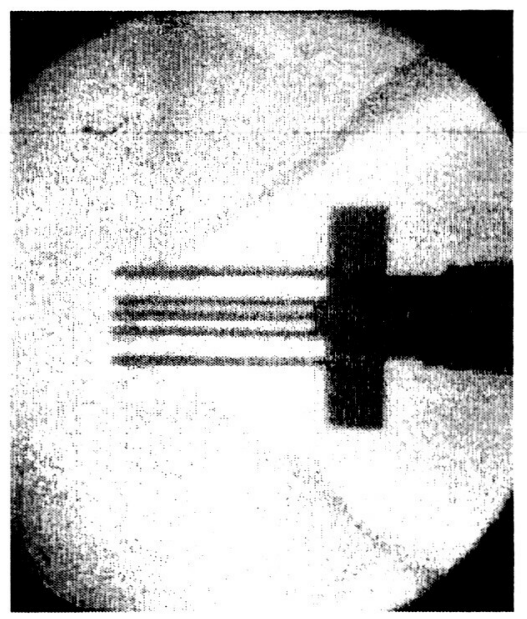

e)-n=5, L/D =1, d/D =0.05

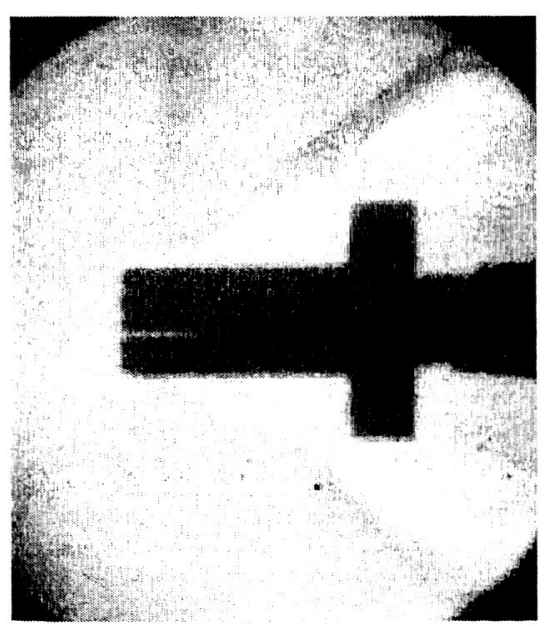

h) $-\mathrm{n}=5, \mathrm{~L} / \mathrm{D}=1, \mathrm{~d} / \mathrm{D}=0.1$

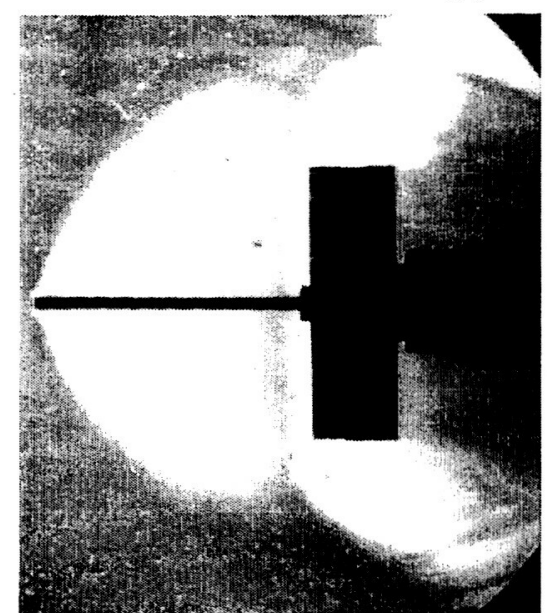

c)-n=1, $L / D=1, d / D=0.05$

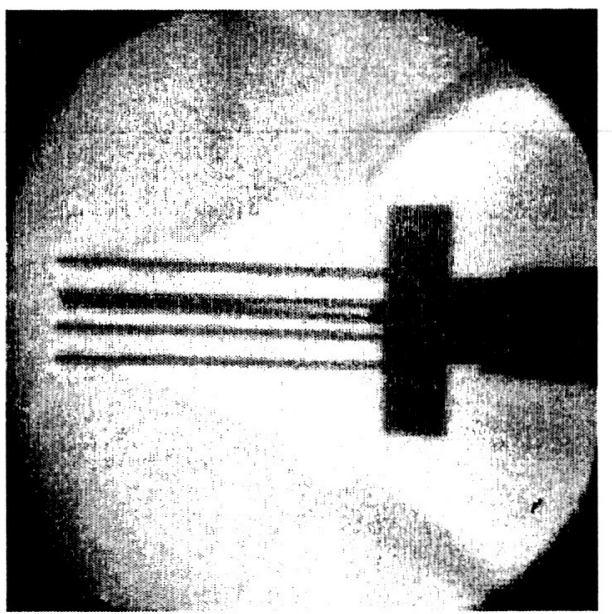

f)-n=5, L/D=1.5, d/D=0.05

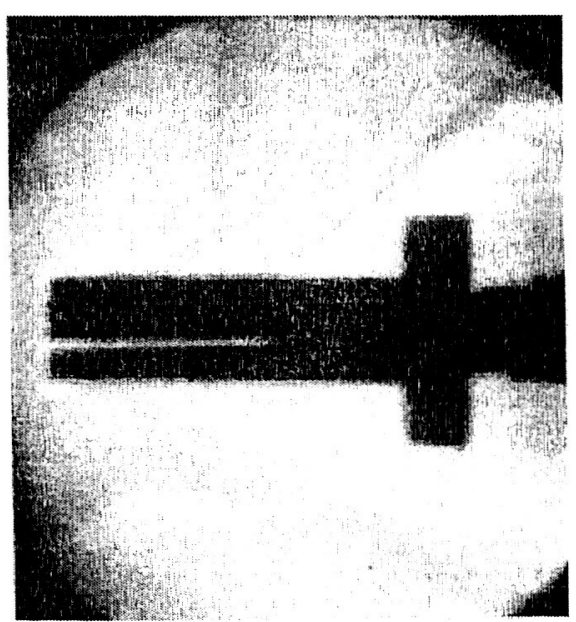

i) $-\mathrm{n}=5, \mathrm{~L} / \mathrm{D}=1.5, \mathrm{~d} / \mathrm{D}=0.1$

Fig.4 1- and 5-needles butt-end models tested in the IM/MSU Wind Tunnel A-7. 


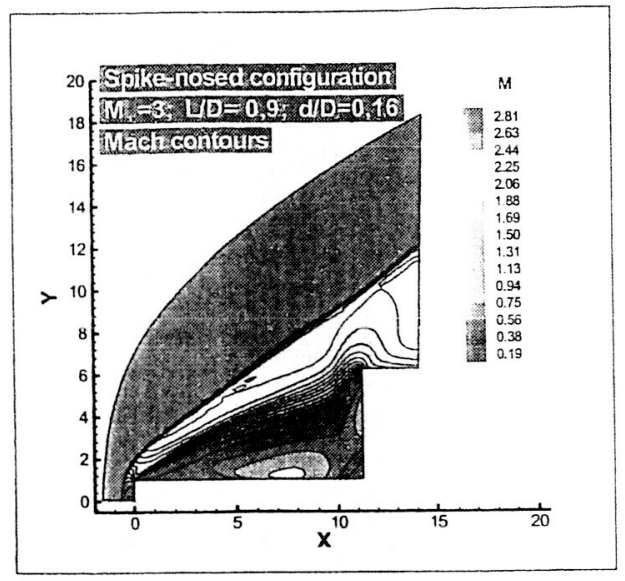

a)

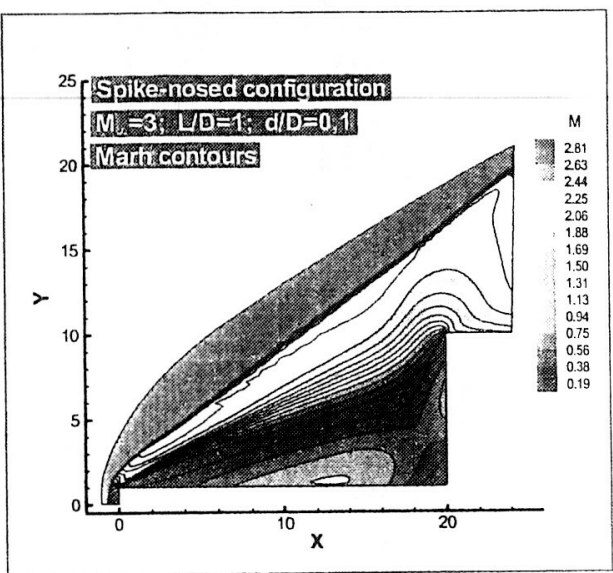

b)

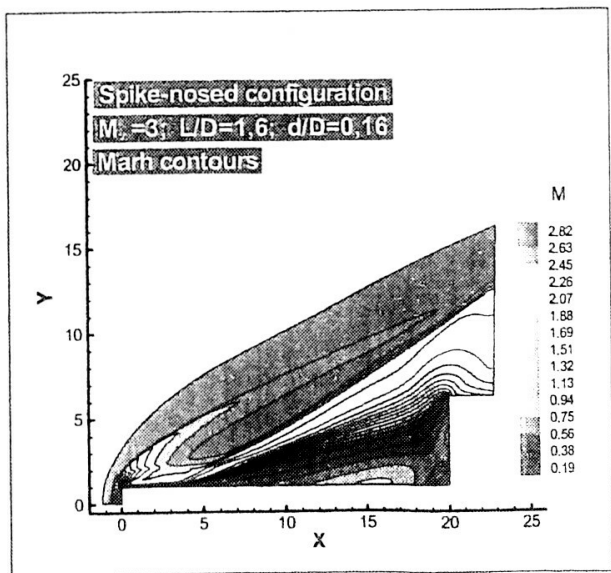

c)

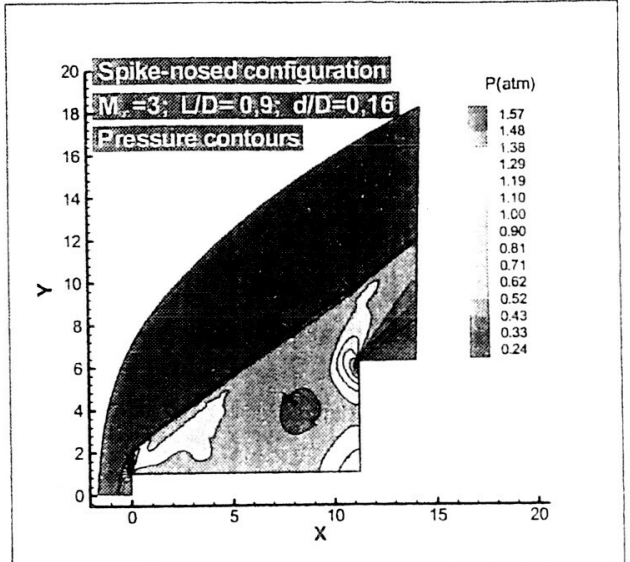

d)

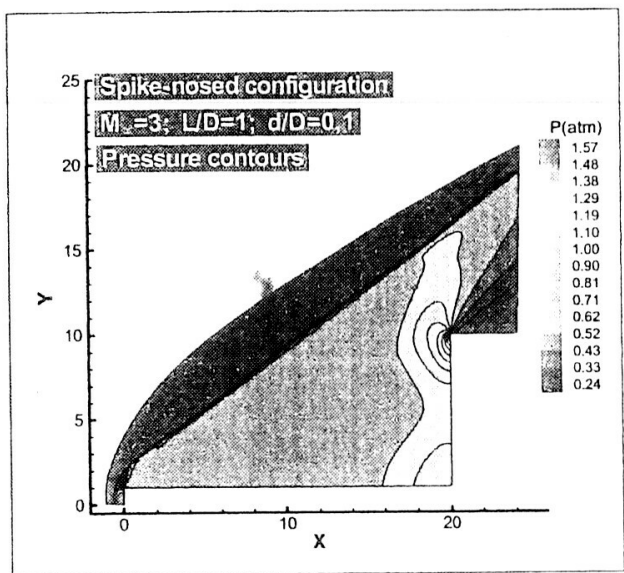

e)

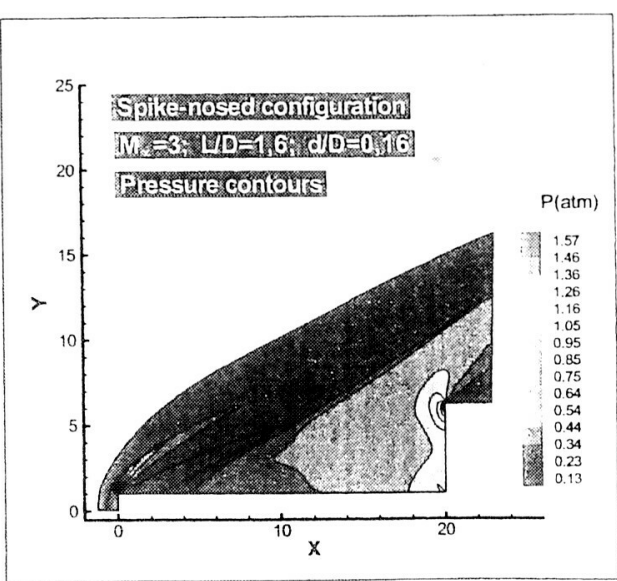

1)

Fig.5 Numerical simulation results based on NSE with $\mathrm{k}-\omega$ turbulence model.

Mach and pressure contours. $M_{\infty}=3 ; d / D=1.6 ; L / D=0.9,1.0,1.6$ 

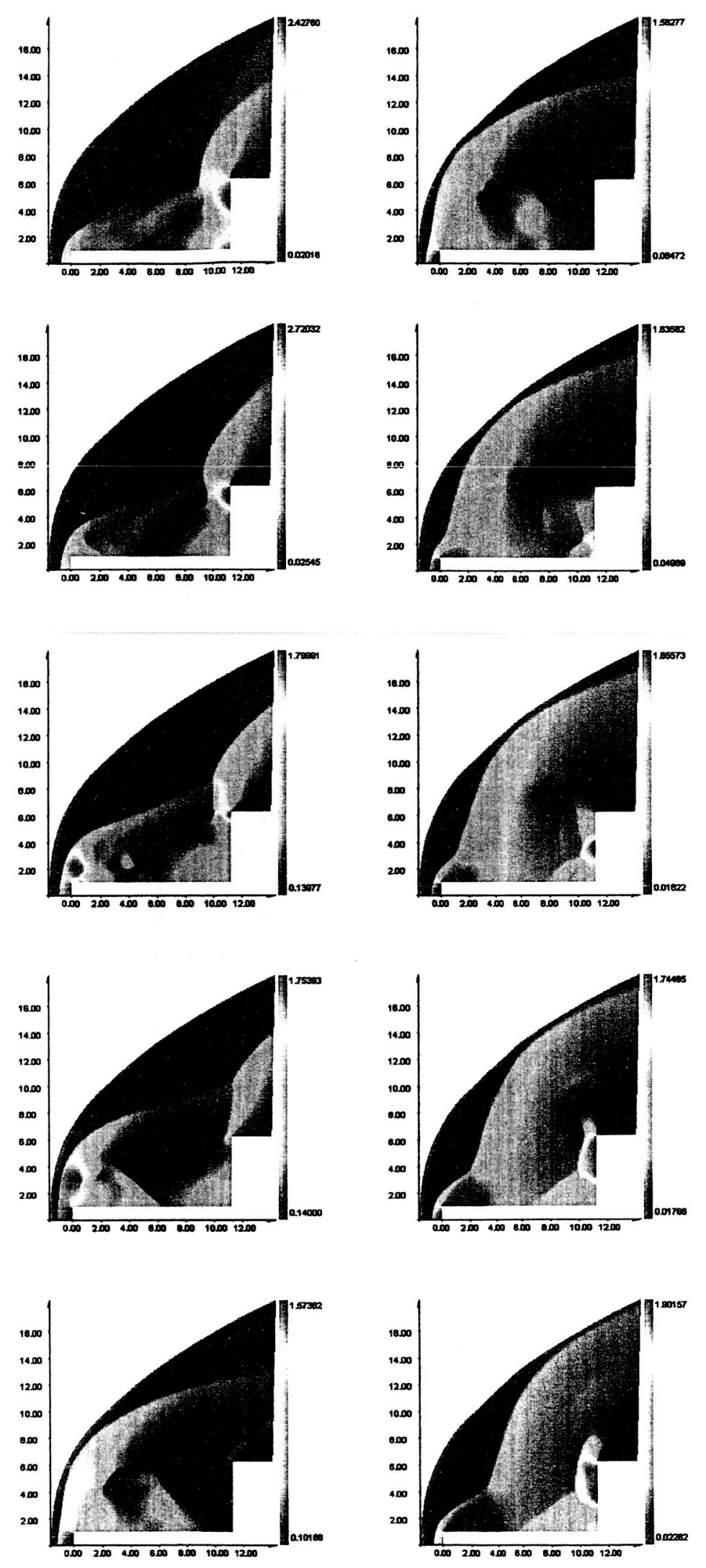
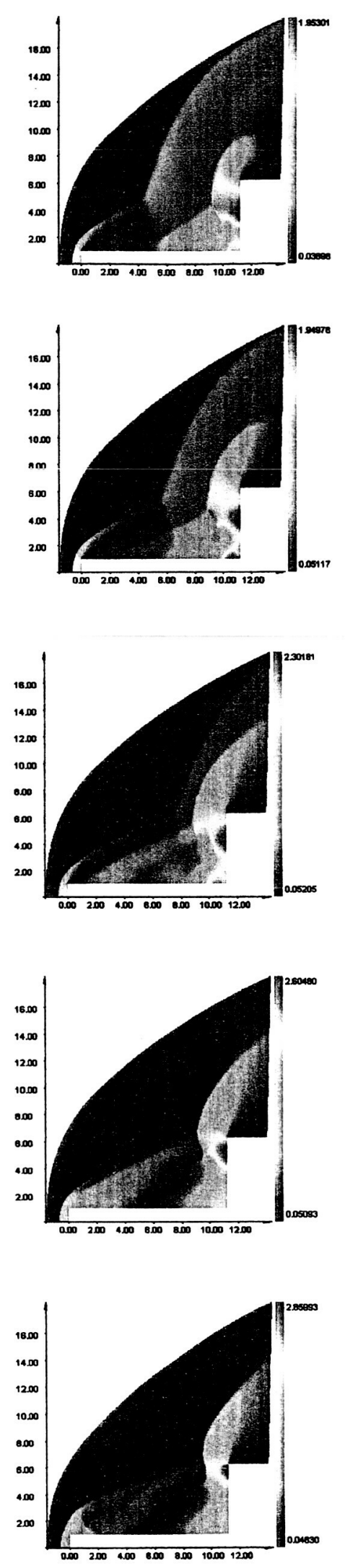

Fig.6 Supersonic flow around butt-end with single needle against the flow. Pressure contours. Oscillatory regime: $M=3 ; T=102^{\circ} \mathrm{K} ; \mathrm{P}=0.14 \mathrm{~atm} ; \operatorname{Re} \sim 1.8$.106 